Radiologe 2014 · 54:964-965

DOI 10.1007/s00117-014-2686-7

Online publiziert: 28. September 2014

(c) Springer-Verlag Berlin Heidelberg 2014

S. Delorme - G. Leidig-Bruckner · T. Wilhelm

Deutsches Krebsforschungszentrum (DKFZ), Heidelberg

\title{
Endokrine Tumoren und ihre Therapie
}

Sehr geehrte Leserinnen und Leser,

das klinische Erscheinungsbild endokriner Tumoren ist vielfältig und wird wesentlich durch Veränderungen im Hormonstoffwechsel geprägt. Weiterhin hängt es vom betroffenen endokrinen Organ, der unterschiedlichen Dignität und teilweise hereditär vorkommenden Erkrankungsformen ab. Daher hat die Familienanamnese einen besonderen Stellenwert sowohl bei der Diagnostik als auch der Therapie endokriner Tumoren. Einige Beispiele:

In einer Familie häufen sich Tumorerkrankungen. Die Tochter hat Schilddrüsenkrebs, der Onkel hatte es auch. Nun wird der Vater beim Fußballtraining im Bauch getroffen, kollabiert und stirbt noch in der Notaufnahme ohne wesentliche Vorerkrankungen. Ursache: Ein Phäochromozytom im Rahmen einer multiplen endokrinen Neoplasie (MEN2a).

Nicht so dramatisch ist der Verlauf eines 50-jährigen Mannes, der beim 30-jährigen Abituriententreffen angesprochen wird, wie sehr sich sein Gesicht mit wulstiger Stirn und knolliger Nase verändert hat. Ihm selbst und seiner Familie war über die Jahre von Tag zu Tag nichts aufgefallen, nur seine Zuckerkrankheit macht ihm seit einigen Jahren Kummer die weitere Abklärung bestätigt die Verdachtsdiagnose einer Akromegalie.

Bei einem anderen Patienten finden sich seit Jahren immer wiederkehrende Magengeschwüre und im Labor fällt ein leicht erhöhtes Serumkalzium auf. Die weitere Abklärung bestätigt die Verdachtsdiagnose einer multiplen endokrinen Neoplasie (MEN1) mit primärem Hyperparathyreoidismus und Gastrinom.
Eine gutaussehende junge Frau muss sich seit ca. drei Monaten rasieren, nicht nur an den Beinen, nein auch im Gesicht, weiterhin bestehen eine vermehrt neu aufgetretene Akne und ein androgen betonter Haarausfall mit Geheimratsecken. Es finden sich eine Hyperandrogenämie und ein Nebennierentumor.

Ein junger Mann wird psychisch auffällig, Besuche beim Psychotherapeuten sind unergiebig, am Ende in der psychiatrischen Notaufnahme fällt im Routinelabor ein erniedrigter Blutzucker auf und als Ursache wird ein Insulinom festgestellt.

Ein 16-Jähriger wird beim Pädiater wegen verzögerter Pubertät vorstellig - die Gonadotropine sind niedrig. Als sich auch nach weiteren zwei Jahren nichts tut, wird die Pubertät medikamentös eingeleitet, mit Erfolg. Bis der junge Mann kurz darauf wegen Gesichtsfeldausfällen in der Augenklinik vorstellig wird und daraufhin beim Neurochirurgen landet.

Eine Frau im Alter von 58 Jahren fällt beim Check-up durch hohe Transaminasen auf. Im Ultraschall ist die Leber mit Metastasen durchsetzt, ebenso im Röntgen die Lunge. Ein Primärtumor wird nie gefunden, die Biopsie eines Leberherdes ergibt einen undifferenzierten neuroendokrinen Tumor. Die Metastasen wachsen rasch und unaufhaltsam, nach $4 \mathrm{Mo}-$ naten ist die Patientin tot.

Diese Fallbeispiele zeigen, wie vielfältig die klinischen Verläufe bei endokrinen Tumoren sein können. Nicht immer sind die geäußerten Beschwerden so eindeutig bzw. können durch andere Ursachen bedingt sein. Daher ist bei bestehendem klinischem Verdacht auf eine endokrine Störung eine entsprechende Hormondiagnostik notwendig. Durch die Mehr- 
oder Minderproduktion von Hormonen greifen diese in vielfacher Weise in unser Befinden, unsere Psyche, gar unser Aussehen ein. Gerade wenn sich Symptome schleichend entwickeln - akromegale Gesichtszüge, Magenulzera beim Gastrinom, Hirsutismus beim Nebennierenkarzinom, Hypoglykämien beim Insulinom, Pubertas tarda beim Kraniopharyngeom - werden sie nur zu lange als „persönliche Marke“ interpretiert denn als Symptom einer Erkrankung.

In der Diagnostik und Therapie greifen Klinik, Labordiagnostik, Radiologie und Nuklearmedizin in faszinierender Weise ineinander; es kann keiner ohne den Anderen. Mit diesem Themenheft möchten wir Sie zu einem Streifzug durch diese Welt einladen und haben bewusst der Klinik breiten Raum gelassen, da sie dem Thema die Faszination verleiht und entscheidend ist für die Planung einer sinnvollen und zielgerichteten Diagnostik und nachfolgenden Therapie. Zwei wichtige Themen werden Sie vermissen: Die Schilddrüse und die Epithelkörperchen. Beide Themen wurden kürzlich in zwei CME-Artikeln umfassend behandelt, und Sie sind eingeladen, diese als Ergänzung hinzuzuziehen $[1,2]$. So bleibt uns jetzt nur, allen Autoren für ihre hervorragenden Beiträge zu danken und Ihnen spannende Lektüre zu wünschen.

$$
\text { fl Cerols-Brecker }
$$

PD Dr. Gudrun Leidig-Bruckner

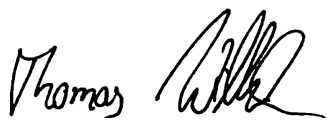

Dr. Thomas Wilhelm

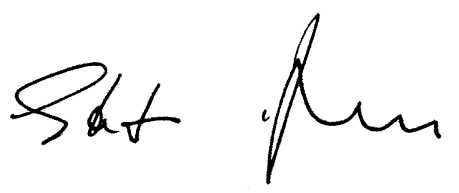

Prof. Dr. Stefan Delorme

\section{Korrespondenzadresse}

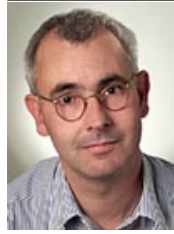

Prof. Dr. S. Delorme

Deutsches Krebsforschungszentrum (DKFZ),

Im Neuenheimer Feld 280,

69120 Heidelberg

s.delorme@dkfz-heidelberg.de

\section{Einhaltung ethischer Richtlinien}

Interessenkonflikt. G. Leidig-Bruckner, T. Wilhelm und S. Delorme geben an, dass kein Interessenkonflikt besteht.

\section{Literatur}

1. Delorme S, Zechmann C, Haberkorn U (2013) Bildgebende Diagnostik des Hyperparathyreoidismus. Radiologe 53:261-276

2. Zechmann C, Haufe 2 (2012) Radiologische und nuklearmedizinische Diagnostik und Therapie der Schilddrüsenerkrankungen. Radiologe 52:761-774

\section{Mit einem Klick in die Welt der Radiologie www.springermedizin.de/radiologie}

Wählen Sie ab sofort den direkten Weg zu Fachbeiträgen und News aus der Radiologie:

Auf www.springermedizin.de/radiologie erwartet Sie ein umfassendes Informations- und Fortbildungsangebot:

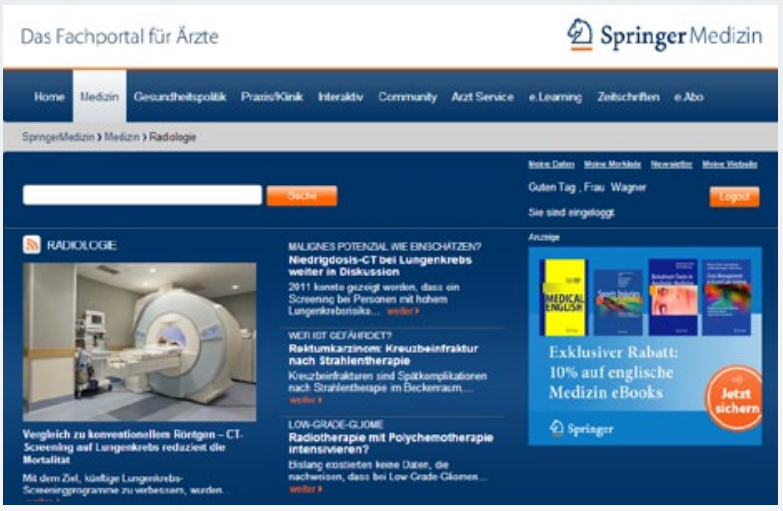

- aktuelle Übersichtsbeiträge und interessante

Kasuistiken aus allen Bereichen der Radiologie

- CME-Beiträge aus den

Fachzeitschriften von Springer Medizin

- kontinuierlich erweiterte Themendossiers

- News aus Berufs- und Gesundheitspolitik

- ausgewählte Meldungen und Beiträge aus der Radiologie

- die wichtigsten Studien und Publikationen -

für Sie zusammengefasst und kommentiert

- Kongress-Highlights

So einfach geht's: Nach einmaliger Registrierung stehen Ihnen zahlreiche Beiträge zur Verfügung.

Unbeschränkten Zugriff auf alle Artikel und Inhalte bietet

e.Med, das Online-Paket von Springer Medizin.

Weitere Infos zu e.Med finden Sie unter

- www.springermedizin.de/eMed 